\title{
Energy-Efficient Transmission Schemes for Cooperative Wireless Powered Cellular Networks
}

\author{
Sun Mao ${ }^{(}$, Student Member, IEEE, Supeng Leng ${ }^{(}$, Member, IEEE, \\ Jie $\left.\mathrm{Hu}^{(}\right)$, Member, IEEE, and Kun Yang ${ }^{\circledR}$, Senior Member, IEEE
}

\begin{abstract}
Wireless powered cellular networks (WPCNs) are promising solution for the future wireless communication systems. This paper proposes a system model of WPCNs, in which the cellular users (CUs) and the device-to-device (D2D) users harvest energy from the hybrid access point (HAP). Some D2D users can help the cell-edge CU to relay its uplink/downlink transmission in exchange for the D2D communication opportunity. For achieving green WPCNs, we formulate two energy efficiency (EE) maximization problems for both uplink and downlink transmissions, respectively. The energy beamformer of the HAP and the time resource allocation are jointly optimized subject to the transmission rate requirements and the available energy constraints of CUs and D2D users. Based on the fractional programming theory and semi-definite relaxation (SDR) method, we transform the originally non-convex EE maximization problems into the equivalent convex problems. This allow us to develop the resource allocation algorithm toward global optimization. The optimal solution in semi-closed form is derived based on Lagrangian method. Extensive simulation results are provided to demonstrate the convergence of the proposed iterative algorithm and the EE gain of the proposed system over the other two baselines.
\end{abstract}

Index Terms-Wireless information and power transfer, cooperation transmission, energy efficiency.

\section{INTRODUCTION}

$\mathbf{I}$ $\mathrm{N}$ THE design of the future fifth-generation (5G) wireless networks, energy harvesting is envisioned as a key technique to enable the perpetual lifetime for wireless devices and cut the last wire [1]-[4]. Harvesting power from renewable sources, such as solar and wind, is a viable solution to prolong the operation lifetime of wireless devices [5]-[7]. However, the

Manuscript received September 15, 2018; revised December 16, 2018; accepted March 6, 2019. Date of publication March 13, 2019; date of current version May 16, 2019. This work was supported in part by the Natural Science Foundation of China under Grant U1705263 and Grant 61601097, in part by the Fundamental Research Funds for the Central Universities, China, under Grant ZYGX2016Z011, in part by the Joint Fund of the Ministry of Education of China and China Mobile under Grant MCM20160304, in part by the Zhongshan City Team Project under Grant 180809162197874, and in part by 111 Project under Grant B14039. The associate editor coordinating the review of this paper and approving it for publication was H. Zhang. (Corresponding author: Supeng Leng.)

S. Mao, S. Leng, and J. Hu are with the School of Information and Communication Engineering, University of Electronic Science and Technology of China, Chengdu 611731, China (e-mail: sunmao@std.uestc.edu.cn; spleng@uestc.edu.cn; hujie@uestc.edu.cn).

$\mathrm{K}$. Yang is with the University of Electronic Science and Technology of China, Chengdu 611731, China, also with Zhongshan Institute, Zhongshan, China, and also with the School of Computer Science and Electronic Engineering, University of Essex, Colchester CO4 3SQ, U.K. (e-mail: kunyang@essex.ac.uk).

Digital Object Identifier 10.1109/TGCN.2019.2904794 unstable nature of renewable energy harvesting may hinder its deployment for various applications with strict quality of experience $(\mathrm{QoE})$ requirements in the future $5 \mathrm{G}$ wireless networks. On the other hand, the radio frequency (RF) signal aided wireless power transfer (WPT) has been envisioned as a promising technique to provide stable and controllable energy supply for wireless devices in the upcoming 5G era [8]-[11]. In a wireless powered cellular network (WPCN), the mobile devices are capable of harvesting energy from the RF signal transmitted by the hybrid access point (HAP) for powering their own information communications [12]-[15]. Nevertheless, the performance of the WPCN is fundamentally restricted by the low power transmission efficiency and the short transmission range [16]-[19].

In order to overcome these limits in the cooperative networks, [20] proposed a harvest-then-cooperate protocol, where a single-antenna HAP broadcast the energy to the source and the relays, then the source and relay pair may upload the information to the HAP by exploiting the energy harvested from the HAP. This model was then extended to the multi-antenna scenario [21], where the HAP is equipped with multiple antennas for improving the system performance. With the aid of the relay, the coverage of the WPCN can be substantially extended. However, a pair of critical drawbacks are observed in the literature related to the cooperative WPCN. First, it is costly to deploy the dedicated relay station for assisting the information transmission. By contrast, user cooperation is an efficient way to improve the information transmission performance [22]. However, mobile terminals sometimes are reluctant to consume its own energy for forwarding other's information. Therefore, it is necessary to design an incentive scheme for encouraging the beneficial user cooperation. Second, most of the existing works focus on optimizing the wireless powered uplink information transmission but ignore the downlink scenario.

Although the optimization of spectrum efficiency becomes the main trend in the previous studies on cooperative WPCNs [21]-[24], the energy efficiency (EE) evaluated by the unit of the bits-per-joule is another performance indicator [25]-[27] for the future wireless networks, especially for the WPT-based systems. Reference [28] investigates the network energy efficiency (EE) maximization of a conventional WPCN. The same group of authors further study the user-centric EE optimization in [29]. Zhou et al. [30] concentrate on energy-efficient power control and spectrum resource allocation in wireless powered device to device 
(D2D) underlay networks. By exploiting stochastic geometry method and optimization theory, Shang et al. [31] study the EE of a wireless-powered D2D-assisted offloading network from a system-level perspective. However, the maximum achievable EE of the cooperative WPCN has not been investigated yet. In particular, it is very difficult to optimize the system EE of cooperative WPCN by jointly developing the cooperation scheme and the resource allocation algorithm.

In order to fill this gap, this paper investigates the energyefficient resource scheduling in a cooperative WPCN. The main contributions of this paper are summarized as follows.

1) User Cooperation Protocol: Different from the existing works, we first propose the user cooperation protocols for both the downlink and the uplink communications in the WPCN, where the users harvest energy from the HAP, and then the selected D2D user consumes a part of harvested power to assist the cell-edge $\mathrm{CU}$ for uplink or downlink information relay so that it can acquire additional time resources from the $\mathrm{CU}$ for its own D2D communication.

2) Joint Energy Beamforming and Time Splitting Optimization Model: The EE of the downlinks in the cooperative WPCN and that of the uplink counterpart can be maximized, respectively, by jointly optimizing the beamformer of the multi-antenna assisted HAP, the resource allocation in the time domain, and the division of the harvested energy between $\mathrm{CU}$ information forwarding and own transmission at the D2D pair, while satisfying the user transmission rate requirement as well as the energy casuality constraint.

3) Global Optimal Resource Allocation Strategy: By exploiting the Dinkelbach's algorithm and the semi-definite relaxation (SDR) method, we transform the original nonconvex EE maximization problems into the convex ones, which allow us to design the resource allocation algorithm to obtain the global optimal solution. In order to get more insights, we further derive the optimal solution in semi-closed form by exploiting the Lagrangian method.

4) Improvement of Energy Efficiency: The simulation results demonstrate that the proposed energy-efficient resource allocation algorithms converges to the optimal solution within a few iterations. Furthermore, the proposed EE-optimal cooperative WPCN outperforms both the throughput-optimal counterpart and the cooperative WPCN with single-antenna HAP, in terms of energy efficiency.

The rest of the paper is organized as follows. Section II presents the related work. In Section III, we introduce the system model and explain the uplink and downlink cooperation protocols. In Sections IV and V, we maximize the EE of the downlink and that of the uplink in the cooperative WPCN, respectively. Simulation results are provided in Section VI, followed by the conclusion of Section VII.

\section{SySTEM MOdEL}

As shown in Fig. 1, this paper considers a typical wireless powered cellular network (WPCN), where a pair of D2D transmitter (DT) and D2D receiver (DR) coexists with a cellular user (CU) via spectrum overlaying [32], [33], i.e., the

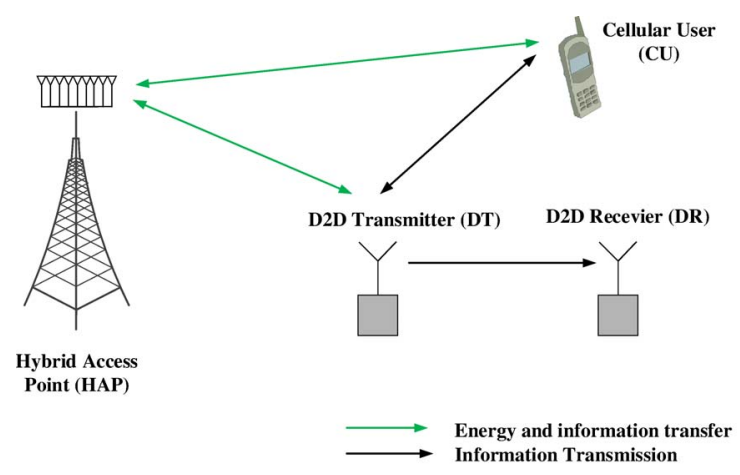

(a)

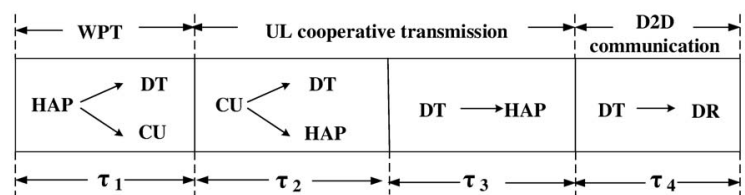

(b)

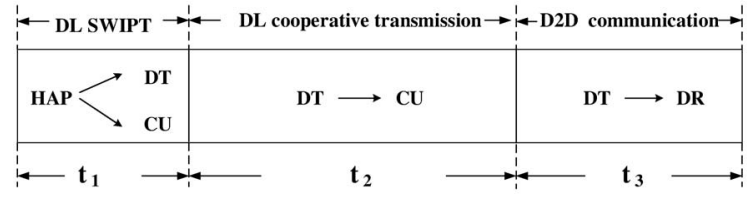

(c)

Fig. 1. System model. (a) Wireless powered cellular networks. (b) Uplink cooperation protocol. (c) Downlink cooperation protocol.

DT can transmit the information to the DR, only if the CU finishes its transmission. The HAP is connected to the power grid for obtaining the stable energy supply. By contrast, all the users are equipped with rechargeable batteries and they can charge their batteries by harvesting the energy from the downlink signal transmitted by the HAP [12].

In this paper, we focus on a scenario that the $\mathrm{CU}$ is far away from the HAP. Therefore, the direct link between the HAP and the CU fails to satisfy the transmission requirement of the $\mathrm{CU}$. As a result, the $\mathrm{CU}$ has to seek for the assistance from the D2D pair in order to successfully transmit/receive the information to/from the remote HAP. For the sake of relaying the information, the DT consumes a certain energy harvested from the HAP to assist the uplink/downlink information transmission of the CU. When the communication requirement of the CU is satisfied, the DT can use the remaining duration of the time block to transmit its own information to the DR. Clearly, the CU can benefit from the cooperation due to the shorter communication range compared to the direct communication with the HAP. Meanwhile, although the DT consumes energy on helping the $\mathrm{CU}$, the longer transmission time can compensate its transmission rate loss caused by the cooperation. This is because the gain from the cooperation transmission reduces the CU's transmission time and allows the HAP to allocate more time for information transmission instead of energy transfer.

\section{A. Channel Model}

It is assumed that the HAP is equipped with $N$ antennas to transmit energy and infomation signal in the downlink and 
TABLE I

SUMmARY OF KEY NOTATIONS

\begin{tabular}{|l|l|}
\hline Notation & Description \\
\hline $\mathbf{h}_{C} / \mathbf{h}_{D}$ & $\begin{array}{l}\text { Downlink channel coefficient between } \\
\text { HAP and CU/DT }\end{array}$ \\
\hline $\mathbf{g}_{C H} / \mathbf{g}_{D H}$ & $\begin{array}{l}\text { Uplink Channel coefficient between } \\
\text { CU/DT and HAP }\end{array}$ \\
\hline $\mathbf{g}_{D D} / \mathbf{g}_{C D}$ & $\begin{array}{l}\text { Channel coefficient between DT and } \\
\text { DR/CU }\end{array}$ \\
\hline$\tau_{i} / t_{i}$ & $\begin{array}{l}\text { Time duration for the scenario of } \\
\text { downlink/uplink }\end{array}$ \\
\hline $\mathbf{w}_{0} / \mathbf{w}_{1}$ & $\begin{array}{l}\text { Energy beamforming for the scenario of } \\
\text { downlink/uplink }\end{array}$ \\
\hline$\eta$ & Energy conversion efficiency \\
\hline$P_{D}$ & Transmission power of DT \\
\hline$\rho$ & Power splitting ratio \\
\hline$P_{H, \max }$ & Maximum transmission power at HAP \\
\hline$r_{c} / r_{d d}$ & $\begin{array}{l}\text { Minimum transmission rate requirement } \\
\text { of CU/DT }\end{array}$ \\
\hline$\eta_{E E}^{u} / \eta_{E E}^{d}$ & Uplink/Downlink energy efficiency \\
\hline
\end{tabular}

only a single antenna is conceived for uplink information reception. In addition, the other terminals are equipped with a single antenna for either the energy harvesting or the information transmission. All channels in our system obey the quasi-static flat-fading, where the channel state remains constant during each time block, but possibly varying from one time block to another [16]. The channel coefficients of the downlinks between the HAP and the CU/DT are denoted by the complex vectors $\mathbf{h}_{C} \in \mathbb{C}^{N \times 1}$ and $\mathbf{h}_{D} \in \mathbb{C}^{N \times 1}$, respectively. Let $\mathbf{g}_{C H}, \mathbf{g}_{D H}, \mathbf{g}_{C D}$, and $\mathbf{g}_{D D}$ denote the uplink channels between the CU and the HAP, that between the DT and the HAP, that between the CU and the DT, as well as that between the DT and the DR, respectively. We assume that the HAP can obtain the global channel state information (CSI) to act as a central controller for promoting cooperation between D2D and cellular users [20]. The key notations are summarized in TABLE I for easy reference.

Note that in the scenario with multiple cell-edge CUs and multiple D2D pairs, the D2D pair is prioritized to forward the cell-edge CU's information with larger $\min \left\{\left|\mathbf{g}_{D H}\right|^{2},\left|\mathbf{g}_{C D}\right|^{2}\right\}$. Intuitively, it can achieve the transmission rate requirement of the cell-edge $\mathrm{CU}$, meanwhile without consuming excessive energy of the D2D pair. In this paper, we focus on the energyefficient cooperation protocol design and resource allocation in a WPCN with single CU and single D2D pair. The methodology can be directly extended to the scenario with multiple CUs and multiple D2D pairs.

\section{B. Uplink Cooperation Protocol}

The cooperation protocol for the uplink communication is illustrated in Fig. 1(b). Each time block is divided into four phases, with time durations of $\tau_{1}, \tau_{2}, \tau_{3}$ and $\tau_{4}$, respectively. Next, we will discuss the four phases of the uplink cooperation protocol.
1) Phase One: The HAP broadcasts the deterministic energy signal to the CU and the DT. Hence, the energy harvested by the CU and DT are calculated as

$$
E_{C}=\eta \tau_{1} \mathbf{w}_{0}^{H} \mathbf{H}_{C} \mathbf{w}_{0}
$$

and

$$
E_{D}=\eta \tau_{1} \mathbf{w}_{0}^{H} \mathbf{H}_{D} \mathbf{w}_{0},
$$

respectively, where we have $\mathbf{H}_{C}=\mathbf{h}_{C} \mathbf{h}_{C}^{H}, \mathbf{H}_{D}=\mathbf{h}_{D} \mathbf{h}_{D}^{H}$, while $\eta \in(0,1]$ denotes the energy conversion efficiency assumed equal for all energy-harvesting devices, and $\mathbf{w}_{0} \in$ $\mathbb{C}^{N \times 1}$ is the downlink beamforming vector of the HAP. In Eqs. (1) and (2), the noise power is negligibly low, when compared to the power of the received signal [34].

2) Phase Two and Three: After the phase of WPT, the CU and DT cooperatively upload the CU's information to the HAP. Specifically, in phase two, the CU broadcasts information to both the DT and HAP. We assume that the users may deplete all the harvested energy for powering the information transmission [29], [35]. Let $N_{0}$ denote the noise power at all receivers. Thus, the information transmission rate of the direct CU-HAP link is expressed as

$$
R_{C H}=\tau_{2} \log \left(1+\frac{\eta\left|\mathrm{g}_{C H}\right|^{2} \tau_{1} \mathbf{w}_{0}^{H} \mathbf{H}_{C} \mathbf{w}_{0}}{\tau_{2} N_{0}}\right) .
$$

In the third phase, the DT decodes the information transmitted by the CU and then forward the decoded information to the HAP. According to the principle of decode-and-forward relaying [3], the cooperative uplink rate of the $\mathrm{CU}$ can be formulated as

$$
R_{C}^{u}=\min \left\{R_{C H}+R_{D H}, R_{C D}\right\},
$$

where $R_{D H}$ and $R_{C D}$ denote the achievable rate of the transmission from the DT to the HAP and that of the transmission from the $\mathrm{CU}$ to the DT, respectively. The transmission rates $R_{D H}$ and $R_{C D}$ can be formulated as

$$
\begin{aligned}
& R_{D H}=\tau_{3} \log \left(1+\frac{\left|\mathrm{g}_{D H}\right|^{2} P_{D}^{(3)}}{N_{0}}\right), \\
& R_{C D}=\tau_{2} \log \left(1+\frac{\eta\left|\mathrm{g}_{C D}\right|^{2} \tau_{1} \mathbf{w}_{0}^{H} \mathbf{H}_{C} \mathbf{w}_{0}}{\tau_{2} N_{0}}\right),
\end{aligned}
$$

respectively, where $P_{D}^{(i)}, i \in\{3,4\}$ represents the transmission power of the DT during the $i$-th phase.

3) Phase Four: After the cooperation phase, the DT transmit its own information to the DR. The rate achieved by the DT in its D2D transmission can be expressed as

$$
R_{D D}=\tau_{4} \log \left(1+\frac{\left|g_{D D}\right|^{2} P_{D}^{(4)}}{N_{0}}\right) .
$$

The uplink energy efficiency is defined as the ratio of the total information rate to the corresponding total energy consumption of the system, which is expressed as

$$
\eta_{E E}^{u}=\frac{R_{C}^{u}+R_{D D}}{\tau_{1}\left\|\mathbf{w}_{0}\right\|^{2}} .
$$




\section{Downlink Cooperation Protocol}

The downlink cooperation protocol is portrayed in Fig. 1(c). Each time block is divided into three phases having the time durations of $t_{1}, t_{2}$, and $t_{3}$, respectively. In the first phase, the HAP broadcasts the modulated signal to both the CU and the DT. Thus, the information rate of the direct HAP-CU is expressed as

$$
R_{H C}=t_{1} \log \left(1+\frac{\mathbf{w}_{1}^{H} \mathbf{H}_{C} \mathbf{w}_{1}}{N_{0}}\right),
$$

where $\mathbf{w}_{1}$ denotes the transmit beamforming vector from the HAP to users. The DT adopts a fixed power splitting scheme having portion $\rho$ of the received signal for the energy harvesting, while having the rest of the received signal for the information decoding. Therefore, the harvested energy and the achievable rate of the HAP-DT link are formulated as

$$
\begin{aligned}
E_{D} & =\eta \rho t_{1} \mathbf{w}_{1}^{H} \mathbf{H}_{D} \mathbf{w}_{1}, \\
R_{H D} & =t_{1} \log \left(1+\frac{(1-\rho) \mathbf{w}_{1}^{H} \mathbf{H}_{D} \mathbf{w}_{1}}{N_{0}}\right),
\end{aligned}
$$

respectively.

In the second phase, the DT decodes the downlink information from the HAP and then forwards the decoded information to the $\mathrm{CU}$ by exploiting the harvested energy. As a result, the achievable rate of cooperative downlink transmission can be obtained as

$$
R_{C}^{d}=\min \left\{R_{H C}+R_{D C}, R_{H D}\right\},
$$

where $R_{D C}$ represent the achievable rate of the transmission from the DT to the $\mathrm{CU}$, which is expressed as

$$
R_{D C}=t_{2} \log \left(1+\frac{\left|g_{C D}\right|^{2} P_{D}^{(2)}}{N_{0}}\right),
$$

where $P_{D}^{(i)}, i \in\{2,3\}$ represents the transmission power of the DT during the $i$-th phase.

After finishing the downlink transmission, the DT transmit its own information to the DR. The rate achieved by the DT is expressed as

$$
R_{D D}=t_{3} \log \left(1+\frac{\left|\mathrm{g}_{D D}\right|^{2} P_{D}^{(3)}}{N_{0}}\right) .
$$

Similar to the uplink scenario, the downlink energy efficiency is then formulated as

$$
\eta_{E E}^{d}=\frac{R_{C}^{d}+R_{D D}}{t_{1}\left\|\mathbf{w}_{1}\right\|^{2}}
$$

Based on the proposed uplink/downlink cooperation protocol, we will discuss the uplink/downlink resource allocation problem in the following sections. Energy efficiency is adopted as the objective function to achieve green cooperative WPCNs.

\section{UPLINK ENERGY EFFICIENCY OPTIMIZATION}

In this section, we aim for maximizing the uplink energy efficiency by jointly optimizing the beamforming vector of the HAP, the time domain resource allocation, and the division of the harvested energy between $\mathrm{CU}$ information forwarding and own transmissions at the D2D user, which can be formulated as

$$
\begin{aligned}
\max _{\boldsymbol{\tau}, \mathbf{w}_{0}, \boldsymbol{P}_{D}} & \eta_{E E}^{u}=\frac{R_{C}^{u}+R_{D D}}{\tau_{1}\left\|\mathbf{w}_{0}\right\|^{2}} \\
\text { s.t. } & \mathrm{C} 1: \sum_{i=1}^{4} \tau_{i} \leq T, \\
& \mathrm{C} 2:\left\|\mathbf{w}_{0}\right\|^{2} \leq P_{H, \max }, \\
& \mathrm{C} 3: \tau_{3} P_{D}^{(3)}+\tau_{4} P_{D}^{(4)} \leq E_{D}, \\
& \mathrm{C} 4: R_{C}^{u} \geq r_{c}, R_{D D} \geq r_{d d}, \\
& \mathrm{C} 5: \tau_{i} \geq 0, i \in\{1,2,3,4\}, \\
& \mathrm{C} 6: P_{D}^{(i)} \geq 0, i \in\{3,4\} .
\end{aligned}
$$

In problem (16), $\mathrm{C} 1$ and $\mathrm{C} 5$ are the time constraints. $\mathrm{C} 2$ represents that the transmit power of the HAP should not exceed its maximum $P_{H, \max }$ during the downlink WPT. C3 indicates the energy causality of the DT, which indicates that the energy used for information transmission should not exceed the energy harvested. In $\mathrm{C} 4, r_{c}$ and $r_{d d}$ denote the minimum transmission rate requirement of the $\mathrm{CU}$ and that of the DT, respectively. C6 guarantees the positive solution for optimal transmit power.

\section{A. Problem Transformation and Solution}

Due to the non-convexity of problem (16), it is very difficult to tackle. By relying on the variable substitution, we equivalently transform the original problem (16) into a more tractable form. Then, the Dinkelbach-type algorithm is adopted for designing an iterative algorithm for solving the transformed fractional optimization problem. Due to the rank-one constraint, the inner-layer optimization problem is NP-hard. The semi-definite relaxation method is then applied to solve this NP-hard problem.

The coupled time variables and power variables make it challenging to solve (16) in its current form. Let us introduce $\mathbf{v}_{0}=\sqrt{\tau_{1}} \mathbf{w}_{0}, \varepsilon_{D}^{(3)}=\tau_{3} P_{D}^{(3)}$ and $\varepsilon_{D}^{(4)}=\tau_{4} P_{D}^{(4)}$. Then, the problem (16) can be reformulated as

$$
\begin{aligned}
& \max _{\boldsymbol{\tau}, \mathbf{v}_{0}, \varepsilon_{D}, R_{C}^{u}} \frac{R_{C}^{u}+\tau_{4} \log \left(1+\frac{\left|\mathrm{g}_{D D}\right|^{2} \varepsilon_{D}^{(4)}}{\tau_{4} N_{0}}\right)}{\left\|\mathbf{v}_{0}\right\|^{2}} \\
& \text { s.t. } \quad \mathrm{C} 7:\left\|\mathbf{v}_{0}\right\|^{2} \leq \tau_{1} P_{H, \max }, \\
& \mathrm{C} 8: \varepsilon_{D}^{(3)}+\varepsilon_{D}^{(4)} \leq \eta \mathbf{v}_{0}^{H} \mathbf{H}_{D} \mathbf{v}_{0}, \\
& \text { C9: } R_{C}^{u} \leq R_{C H}+R_{D H} \\
&=\tau_{2} \log \left(1+\frac{\eta\left|g_{C H}\right|^{2} \mathbf{v}_{0}^{H} \mathbf{H}_{C} \mathbf{v}_{0}}{\tau_{2} N_{0}}\right) \\
&+\tau_{3} \log \left(1+\frac{\left|\mathrm{g}_{D H}\right|^{2} \varepsilon_{D}^{(3)}}{\tau_{3} N_{0}}\right),
\end{aligned}
$$




$$
\begin{aligned}
& \mathrm{C} 10: R_{C}^{u} \leq R_{C D}=\tau_{2} \log \left(1+\frac{\eta\left|\mathrm{g}_{C D}\right|^{2} \mathbf{v}_{0}^{H} \mathbf{H}_{C} \mathbf{v}_{0}}{\tau_{2} N_{0}}\right), \\
& \mathrm{C} 1, \mathrm{C} 4-\mathrm{C} 5 .
\end{aligned}
$$

Then, we exploit the fractional structure of the EE expression to reformulate the problem (17):

$$
\begin{array}{cl}
\max _{\boldsymbol{\tau}, \mathbf{v}_{0}, \boldsymbol{\varepsilon}_{D}, R_{C}^{u}} & R_{C}^{u}+\tau_{4} \log \left(1+\frac{\left|\mathrm{g}_{D D}\right|^{2} \varepsilon_{D}^{(4)}}{\tau_{4} N_{0}}\right)-\eta_{E E}^{*}\left\|\mathbf{v}_{0}\right\|^{2} \\
\text { s.t. } & \mathrm{C} 1, \mathrm{C} 4-\mathrm{C} 5, \mathrm{C} 7-\mathrm{C} 10 .
\end{array}
$$

where $\eta_{E E}^{*}$ is the optimal $\mathrm{EE}$ of the resource allocation problem (17). According to [26], Dinkelbach's algorithm can be applied to obtain global optimal $\eta_{E E}^{*}$. The key of this algorithm is to alternately solve the following optimization problem:

$$
\begin{aligned}
& \max _{\boldsymbol{\tau}, \mathbf{V}_{0}, \varepsilon_{D}, R_{C}^{u}} R_{C}^{u}+\tau_{4} \log \left(1+\frac{\left|\mathrm{g}_{D D}\right|^{2} \varepsilon_{D}^{(4)}}{\tau_{4} N_{0}}\right)-u \operatorname{Tr}\left(\mathbf{V}_{0}\right) \\
& \text { s.t. } \mathrm{C} 11: \operatorname{Tr}\left(\mathbf{V}_{0}\right) \leq \tau_{1} P_{H, \text { max }} \text {, } \\
& \text { C12: } \varepsilon_{D}^{(3)}+\varepsilon_{D}^{(4)} \leq \eta \operatorname{Tr}\left(\mathbf{H}_{D} \mathbf{V}_{0}\right), \\
& \text { C13: } R_{C}^{u} \leq R_{C H}+R_{D H} \\
& =\tau_{2} \log \left(1+\frac{\eta\left|\mathrm{g}_{C H}\right|^{2} \operatorname{Tr}\left(\mathbf{H}_{C} \mathbf{V}_{0}\right)}{\tau_{2} N_{0}}\right) \\
& +\tau_{3} \log \left(1+\frac{\left|\mathrm{g}_{D H}\right|^{2} \varepsilon_{D}^{(3)}}{\tau_{3} N_{0}}\right), \\
& \text { C14: } R_{C}^{u} \leq R_{C D}=\tau_{2} \log \left(1+\frac{\eta\left|g_{C D}\right|^{2} \operatorname{Tr}\left(\mathbf{H}_{C} \mathbf{V}_{0}\right)}{\tau_{2} N_{0}}\right) \text {, } \\
& \mathrm{C} 15: \mathbf{V}_{0} \succeq \mathbf{0} \text {, } \\
& \text { C16: } \operatorname{Rank}\left(\mathbf{V}_{0}\right)=1 \text {, } \\
& \text { C1, C4-C5. }
\end{aligned}
$$

where $\mathbf{V}_{0}=\mathbf{v}_{0} \mathbf{v}_{0}^{H}$ represents the energy transmission covariance matrix, and $u$ is an update parameter. The procedure of the proposed uplink resource allocation algorithm is summarized in Algorithm 1.

The computational complexity of the Algorithm 1 can be divided into two parts, namely the iteration number and the computational complexity in each iteration. According to [26], the Dinkelbach-type algorithm exhibits a super-linear convergence rate. At each iteration of Algorithm 1, the computational complexity of solving rank-relaxation problem (19) is $O\left(\left(a^{2} b+a^{3}\right) b^{\frac{1}{2}}\right)$ [36], where $a=N^{2}+7$ denotes the decision variable and $b=8$ is the constraint.

Furthermore, we discuss the application of the proposed model and approach in a practical wireless powered cellular network. The AP first conducts channel estimation via forward-link training or reverse-link feedback. After obtaining the global channel state information (CSI), the AP then executes the proposed energy-efficient resource allocation algorithm for getting optimal time assignment, energy beamforming design, and energy allocation between $\mathrm{CU}$ information forwarding and own transmission at the D2D pair. Next, the corresponding resource allocation parameters are broadcasted by the AP in the downlink. Finally, the system will operate according the optimal resource allocation parameters.

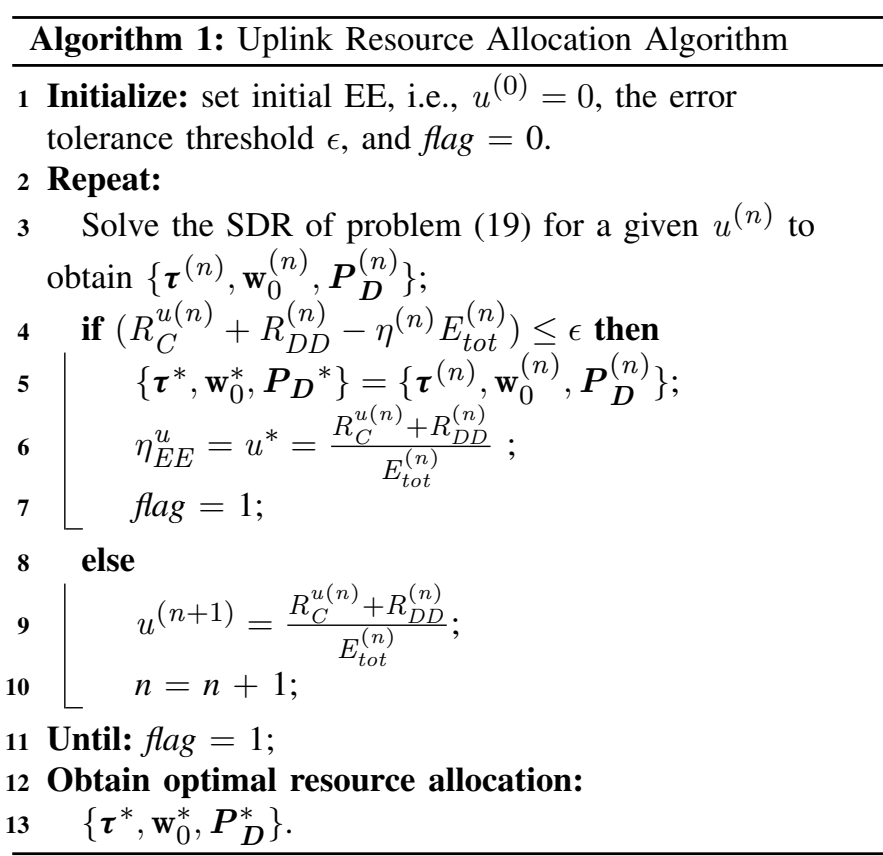

Lemma 1: The proposed Algorithm 1 can converge to the global optimal solution.

Proof: Before the proof, we define

$$
G(u)=\max _{x}\left\{R_{c}^{u}(x)+R_{D D}(x)-u E_{t o t}(x)\right\},
$$

where $x=\left\{\boldsymbol{\tau}, \mathbf{V}_{0}, \boldsymbol{\varepsilon}_{D}, R_{C}\right\}$ denotes the optimization variable set of the problem (19). According to [37], the function $G(u)$ has the following characterizations:

- $G(u)$ is monotonically decreasing.

- For any $\bar{x}$ and $u_{\bar{x}}=\frac{R_{c}^{u}(\bar{x})+R_{D D}(\bar{x})}{E_{t o t}(\bar{x})}$, we have $G\left(u_{\bar{x}}\right) \geq 0$, and the equality holds when $\bar{x}=$ $\underset{x}{\operatorname{argmax}}\left\{R_{c}^{u}(x)+R_{D D}(x)-u_{\bar{x}} E_{t o t}(x)\right\}$.

Given the iteration rule for $u$ in Algorithm 1, we have

$$
\begin{aligned}
G\left(u^{(n)}\right) & =\left\{R_{c}^{u}\left(x^{*(n)}\right)+R_{D D}\left(x^{*(n)}\right)-u^{(n)} E_{t o t}\left(x^{*(n)}\right)\right\} \\
& =\left(u^{(n+1)}-u^{(n)}\right) E_{t o t}\left(x^{*(n)}\right) \geq 0 .
\end{aligned}
$$

Since $E_{\text {tot }}\left(x^{*(n)}\right)>0$, it follows $u^{(n+1)} \geq u^{(n)}$. According to the decreasing property of $G(u)$, we have $G\left(u^{(n+1)}\right) \leq$ $G\left(u^{(n)}\right)$. Integrating with the compactness of the feasible set, thus, Algorithm 1 converges.

Next, we will prove that Algorithm 1 can converge to the global optimal solution by contradiction. It is assumed that the limit point of $\left\{u^{(n)}\right\}$ satisfies $\bar{u}<u^{*}$. Then, we have $G(\bar{u})>$ $G\left(u^{*}\right)$. For the convergence point $\bar{u}=\frac{R_{c}^{u}(\bar{x})+R_{D D}(\bar{x})}{E_{t o t}(\bar{x})}$, we have $\bar{x}=\operatorname{argmax}\left\{R_{c}^{u}(x)+R_{D D}(x)-u_{\bar{x}} E_{t o t}(x)\right\}$. Therefore, $G(\bar{u})=0 . \stackrel{x}{x}$ Meanwhile, we also know $G\left(u^{*}\right)=0$, which is a contradiction.

\section{B. Optimal Solution for Problem (19)}

In this section, we concentrate on solving the problem (19) for a given $u$, and derive its optimal solution via lagrangian 
duality method. By removing the rank constraint C16, the SDR of problem (19) becomes convex, which can be solved by a variety of convex optimization solver, such as MOSEK. Then, we prove the tightness of the relaxation, and further obtain the optimal solution of the problem (19) in semi-closed form by leveraging the lagrangian method.

Theorem 1: The SDR of problem (19) is convex.

Proof: Firstly, we prove the concavity of $\tau_{4} \log (1+$ $\left.\frac{\left|\mathrm{g}_{D D}\right|^{2} \varepsilon_{D}^{(4)}}{\tau_{4} N_{0}}\right)$ over $\tau_{4}$ and $\varepsilon_{D}^{(4)}$. According to [38], if $f(x)$ is a concave function, its perspective function $g(x, t)=t f(x / t)$ is also concave. Obviously, $\log \left(1+\frac{\left|g_{D D}\right|^{2} \varepsilon_{D}^{(4)}}{N_{0}}\right)$ is a concave function of $\varepsilon_{D}^{(4)}$. Thus, its perspective function $\tau_{4} \log \left(1+\frac{\left|\mathrm{g}_{D D}\right|^{2} \varepsilon_{D}^{(4)}}{\tau_{4} N_{0}}\right)$ is a concave function of $\tau_{4}$ and $\varepsilon_{D}^{(4)}$. As a result, the objective function in the problem (19) is convex. Similarly, $R_{C H}$, $R_{D H}, R_{D D}$ and $R_{C D}$ in the problem (19) are also concave functions. Thus, their corresponding constraints $\mathrm{C} 4, \mathrm{C} 13$ and C14 are convex. The other constraints in the SDR of problem (19) are linear with respect to $\left\{\boldsymbol{\tau}, \mathbf{V}_{0}, \boldsymbol{\varepsilon}_{D}, R_{C}\right\}$. Hence, the SDR of problem (19) is proved to be a convex optimization problem.

Theorem 2: If the problem (19) is solvable, there exists an optimal $\mathbf{V}_{0}^{*}$ for the SDR of problem (19) such that $\operatorname{Rank}\left(\mathbf{V}_{0}^{*}\right)=1$.

Proof: See Appendix A.

Theorem 2 declares that the rank constraint relaxation is tight. Therefore, the global optimal solution to the resource allocation problem (16) can always be found by our proposed algorithm. Since the SDR of (19) is convex and satisfies Slater's condition, the strong duality holds. In order to gain more insights, the lagrangian method is applied to derive the optimal solution of problem (19). The lagrangian of (19) is given by

$$
\begin{aligned}
\mathcal{L}_{1}= & R_{C}^{u}+\tau_{4} \log \left(1+\frac{\left|\mathbf{g}_{D D}\right|^{2} \varepsilon_{4}^{(D)}}{\tau_{4} N_{0}}\right)-u \operatorname{Tr}\left(\mathbf{V}_{0}\right) \\
& +a_{1}\left(\tau_{1} P_{H, \max }-\operatorname{Tr}\left(\mathbf{V}_{0}\right)\right) \\
& +a_{2}\left(\eta \operatorname{Tr}\left(\mathbf{H}_{D} \mathbf{V}_{0}\right)-\varepsilon_{D}^{(3)}-\varepsilon_{D}^{(4)}\right) \\
& +a_{3}\left(\tau_{2} \log \left(1+\frac{\eta\left|\mathbf{g}_{C H}\right|^{2} \operatorname{Tr}\left(\mathbf{H}_{C} \mathbf{V}_{0}\right)}{\tau_{2} N_{0}}\right)\right. \\
& \left.+\tau_{3} \log \left(1+\frac{\left|\mathbf{g}_{D H}\right|^{2} \varepsilon_{D}^{(3)}}{\tau_{3} N_{0}}\right)\right) \\
& +a_{4}\left(\tau_{2} \log \left(1+\frac{\eta\left|\mathbf{g}_{C D}\right|^{2} \operatorname{Tr}\left(\mathbf{H}_{C} \mathbf{V}_{0}\right)}{\tau_{2} N_{0}}\right)-R_{C}^{u}\right) \\
& +a_{5}\left(T-\sum_{i=1}^{4} \tau_{i}\right)+a_{6}\left(R_{C}^{u}-r_{C}\right) \\
& +a_{7}\left(\tau_{4} \log \left(1+\frac{\left|\mathbf{g}_{D D}\right|^{2} \varepsilon_{D}^{(4)}}{N_{0} \tau_{4}}\right)-r_{d d}\right)
\end{aligned}
$$

where $a_{1}-a_{7}$ are the non-negative lagrangian multipliers associated with the inequality constraints of (19).
Theorem 3: The optimal solution $\left(\varepsilon_{D}^{*}, \tau^{*}\right)$ of (19) is given by

$$
\begin{aligned}
\varepsilon_{D}^{(3) *} & =\tau_{3}^{*}\left[\frac{a_{3}^{*}}{a_{2}^{*} \ln 2}-\frac{N_{0}}{\left|\mathbf{g}_{D H}\right|^{2}}\right]^{+}, \\
\varepsilon_{D}^{(4) *} & =\tau_{4}^{*}\left[\frac{1+a_{7}^{*}}{a_{2}^{*} \ln 2}-\frac{N_{0}}{\left|\mathbf{g}_{D D}\right|^{2}}\right]^{+}, \\
\tau_{1}^{*} & =\left\{\begin{array}{l}
T-\sum_{i=2} \tau_{i}^{*} \\
\tau_{2}^{*}= \\
\tau_{5}^{*}>0 \\
\left.\tau_{3}^{*}=-\frac{\left.\sum_{i=2} \tau_{i}^{*}\right] a_{5}^{*}=0}{-\eta\left|\mathbf{g}_{C D}\right|^{2} \operatorname{Tr}\left(\mathbf{H}_{C} \mathbf{V}_{0}^{*}\right)}\right) \\
\left.\tau_{4}^{*}=-\frac{1}{\mathcal{W}\left(-\exp \left(-\left(\frac{a_{5}^{*} \ln 2}{a_{3}^{*}+a_{4}^{*}}+1\right)\right)\right)}\right) \\
N_{0}\left(1+\frac{\left|\mathbf{g}_{D H}\right|^{2} \varepsilon_{D}^{(3) *}}{\mathcal{W}\left(-\exp \left(-\left(\frac{a_{5}^{*} \ln 2}{1+a_{7}^{*}}+1\right)\right)\right)}\right)
\end{array},\right.
\end{aligned}
$$

where $[x]^{+}=\max (x, 0)$, and $\mathcal{W}(x)$ is the Lambert $\mathrm{W}$ function defined as the solution for $\mathcal{W}(x) \exp (\mathcal{W}(x))=x$ [39].

Proof: See Appendix B.

\section{Downlink ENERGy EFFICIENCY OptimizATION}

Besides the cooperative uplink transmission, the energyefficient downlink transmission design is another significiant objective for cooperative WPCNs. In this section, we investigate the optimal beamforming and time allocation in order to maximize the system downlink EE, the optimization problem is formulated as:

$$
\begin{array}{cl}
\max _{\boldsymbol{t}, \mathbf{w}_{1}, \boldsymbol{P}_{\boldsymbol{D}}} & \eta_{E E}^{d}=\frac{R_{C}^{d}+R_{D D}}{t_{1}\left\|\mathbf{w}_{1}\right\|^{2}} \\
\text { s.t. } & \text { C1': } \sum_{i=1}^{3} t_{i} \leq T, \\
& \text { C2': }\left\|\mathbf{w}_{1}\right\|^{2} \leq P_{H, \max }, \\
& \text { C3': } t_{2} P_{D}^{(2)}+t_{3} P_{D}^{(3)} \leq E_{D}, \\
& \text { C4': } R_{C}^{d} \geq r_{c}, R_{D D} \geq r_{d d}, \\
& \text { C5': } t_{i} \geq 0, i \in\{1,2,3\}, \\
& \text { C6': } P_{D}^{(i)} \geq 0, i \in\{2,3\},
\end{array}
$$

In problem (29), C1' denotes that the total cooperative transmission duration cannot exceed the time block length $T$. C2' restricts the maximum transmission power $P_{H, \max }$ at the HAP. C3' represents that the consumed energy of D2D user should be not higher than its harvested energy. C4' guarantees the target transmission rate at the CU and D2D user. 
Obviously, the problem (29) is hard non-convex. In the next, we will convert it to an equivalient convex model.

If we use the similar variable substitution as adopted in Section IV-A, i.e., $\mathbf{V}_{1}=t_{1} \mathbf{w}_{1} \mathbf{w}_{1}^{H}, \varepsilon_{D}^{(2)}=t_{2} P_{D}^{(2)}$ and $\varepsilon_{D}^{(3)}=$ $t_{3} P_{D}^{(3)}$, the problem (29) can be rewritten as

$$
\begin{aligned}
& \max _{\boldsymbol{t}, \mathbf{V}_{1}, \boldsymbol{\varepsilon}_{D}, R_{C}^{d}} \frac{R_{C}^{d}+\tau_{3} \log \left(1+\frac{\left|\mathrm{g}_{D D}\right|^{2} \varepsilon_{D}^{(3)}}{t_{3} N_{0}}\right)}{\operatorname{Tr}\left(\mathbf{V}_{1}\right)} \\
& \text { s.t. } \quad \mathrm{C} 7^{\prime}: \operatorname{Tr}\left(\mathbf{V}_{1}\right) \leq t_{1} P_{H, \max }, \\
& \mathrm{C} 8^{\prime}: \varepsilon_{D}^{(2)}+\varepsilon_{D}^{(3)} \leq \eta \rho \operatorname{Tr}\left(\mathbf{H}_{D} \mathbf{V}_{1}\right) \text {, } \\
& \text { C9': } R_{C}^{d} \leq R_{H C}+R_{D C} \\
& =t_{1} \log \left(1+\frac{\operatorname{Tr}\left(\mathbf{H}_{C} \mathbf{V}_{1}\right)}{t_{1} N_{0}}\right) \\
& +t_{2} \log \left(1+\frac{\left|\mathrm{g}_{C D}\right|^{2} \varepsilon_{D}^{(2)}}{t_{2} N_{0}}\right) \text {, } \\
& \text { C10': } R_{C}^{d} \leq R_{H D}=t_{1} \log \left(1+\frac{(1-\rho) \operatorname{Tr}\left(\mathbf{H}_{D} \mathbf{V}_{1}\right)}{t_{1} N_{0}}\right) \text {, } \\
& \text { C11': } \mathbf{V}_{1} \succeq \mathbf{0} \text {, } \\
& \text { C12': } \operatorname{Rank}\left(\mathbf{V}_{1}\right)=1 \text {, } \\
& \text { C1', C4' - C5'. }
\end{aligned}
$$

Due to the fractional structure of the EE, the problem (30) is still non-convex. Based on the Dinkelbach's algorithm, we can obtain the optimal downlink EE by alternating solving the following problem:

$$
\begin{array}{cl}
\max _{\boldsymbol{t}, \mathbf{V}_{1}, \boldsymbol{\varepsilon}_{D}, R_{C}^{d}} & R_{C}^{d}+t_{3} \log \left(1+\frac{\left|\mathrm{g}_{D D}\right|^{2} \varepsilon_{D}^{(3)}}{t_{3} N_{0}}\right)-u \operatorname{Tr}\left(\mathbf{V}_{1}\right) \\
\text { s.t. } & \mathrm{C} 1^{\prime}, \mathrm{C} 4^{\prime}-\mathrm{C} 5 \text { ', } \mathrm{C}^{\prime}{ }^{\prime}-\mathrm{C} 12^{\prime} .
\end{array}
$$

By removing the rank-one constraint $\mathrm{C} 12$ ', the problem (31) is transformed to a convex optimization problem, which can be solved by various solvers. The detailed procedure of the downlink resource allocation algorithm is illustrated in Algorithm 2. Problem (31) is with $c=N^{2}+6$ optimization variables and $d=8$ constraints such that the computational complexity is given by $O\left(\left(c^{2} d+c^{3}\right) d^{\frac{1}{2}}\right)$. Next, we will prove the tightness of the rank relaxation and deduce the optimal solution based on the KKT conditions.

Theorem 4: The SDR of problem (31) is convex.

Proof: The proof is similar to Theorem 1 and is omitted.

Theorem 5: If the problem (31) is solvable, there exists an optimal $\mathbf{V}^{*}$ for the SDR of problem (31) such that $\operatorname{Rank}\left(\mathbf{V}_{1}^{*}\right)=1$.

Proof: See Appendix C.

Theorem 5 proves the tightness of the rank constraint relaxation. Hence, the proposed Algorithm 2 can always find a global optimal solution. For obtaining more insights, the Lagrangian method is used to derive the optimal solution of (31). The Lagrangian of (31) is shown as

$$
\begin{aligned}
\mathcal{L}_{2}= & R_{C}^{d}+t_{3} \log \left(1+\frac{\left|\mathbf{g}_{D D}\right|^{2} \varepsilon_{D}^{(3)}}{t_{3} N_{0}}\right)-u \operatorname{Tr}\left(\mathbf{V}_{1}\right) \\
& +b_{1}\left(T-\sum_{i=1}^{3} t_{i}\right)+b_{2}\left(R_{C}^{d}-r_{c}\right)
\end{aligned}
$$

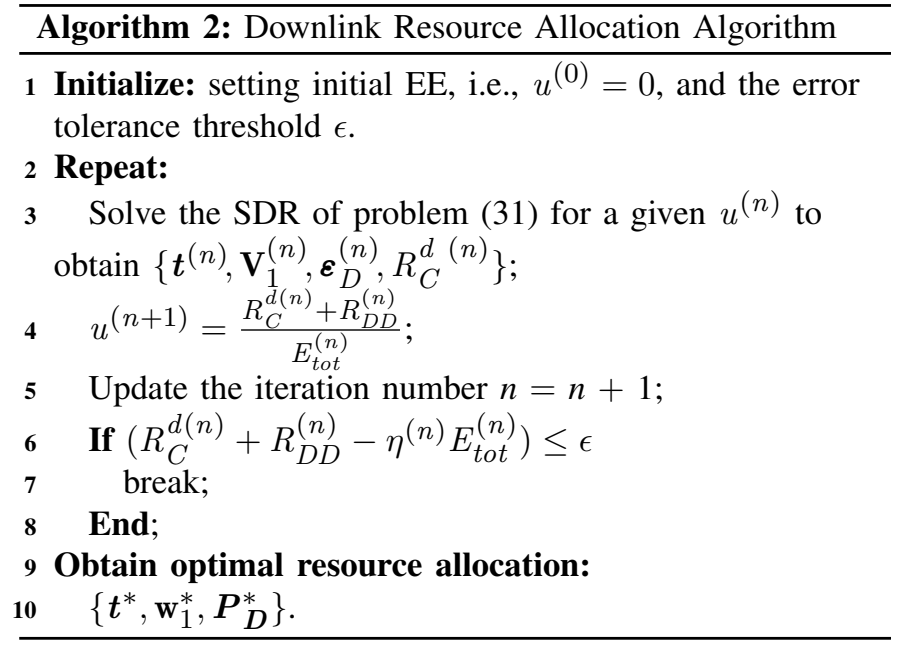

$$
\begin{aligned}
& +b_{3}\left(t_{3} \log \left(1+\frac{\left|\mathbf{g}_{D D}\right|^{2} \varepsilon_{D}^{(3)}}{t_{3} N_{0}}\right)-r_{d d}\right) \\
& +b_{4}\left(t_{1} P_{H, \max }-\operatorname{Tr}\left(\mathbf{V}_{1}\right)\right) \\
& +b_{5}\left(\eta \rho \operatorname{Tr}\left(\mathbf{H}_{D} \mathbf{V}_{1}\right)-\varepsilon_{D}^{(2)}-\varepsilon_{D}^{(3)}\right) \\
& +b_{6}\left(t_{1} \log \left(1+\frac{\operatorname{Tr}\left(\mathbf{H}_{C} \mathbf{V}_{1}\right)}{t_{1} N_{0}}\right)\right. \\
& \left.\quad+t_{2} \log \left(1+\frac{\left|\mathbf{g}_{C D}\right|^{2} \varepsilon_{D}^{(2)}}{t_{2} N_{0}}\right)-R_{c}^{d}\right) \\
& +b_{7}\left(t_{1} \log \left(1+\frac{(1-\rho) \operatorname{Tr}\left(\mathbf{H}_{D} \mathbf{V}_{1}\right)}{t_{1} N_{0}}\right)-R_{c}^{d}\right),
\end{aligned}
$$

where $b_{1}-b_{7}$ are non-negative Lagrangian multipliers.

Theorem 6: The optimal solution $\left(\varepsilon_{D}^{*}, t^{*}\right)$ of $(31)$ is written as

$$
\begin{aligned}
\varepsilon_{D}^{(2) *} & =t_{2}^{*}\left[\frac{b_{6}^{*}}{b_{5}^{*} \ln 2}-\frac{N_{0}}{\left|\mathbf{g}_{C D}\right|^{2}}\right]^{+}, \\
\varepsilon_{D}^{(3) *} & =t_{3}^{*}\left[\frac{1+b_{3}^{*}}{b_{5}^{*} \ln 2}-\frac{N_{0}}{\left|\mathbf{g}_{D D}\right|^{2}}\right]^{+}, \\
t_{1}^{*} & =\left\{\begin{array}{l}
T-\sum_{i=2} t_{i}^{*} b_{1}^{*}>0 \\
\in\left(0, T-\sum_{i=2}^{3} t_{i}^{*}\right] b_{1}^{*}=0
\end{array}\right. \\
t_{2}^{*} & =-\frac{\left|\mathbf{g}_{C D}\right|^{2} \varepsilon_{D}^{(2) *}}{N_{0}+\frac{N_{0}}{\mathcal{W}\left(-\exp \left(-\left(\frac{b_{1}^{*} \ln 2}{b_{6}^{*}}+1\right)\right)\right.}}, \\
t_{3}^{*} & =-\frac{\left|\mathbf{g}_{D D}\right|^{2} \varepsilon_{D}^{(3) *}}{N_{0}+\frac{N_{0}}{\mathcal{W}\left(-\exp \left(-\left(\frac{b_{1}^{*} \ln 2}{1+b_{3}^{*}}+1\right)\right)\right.}} .
\end{aligned}
$$

Proof: See Appendix D.

Lemma 2: The proposed Algorithm 2 can converge to the global optimal solution.

Proof: The proof is similar to the Lemma 1 and is omitted. 
TABLE II

SIMULATION PARAMETERS

\begin{tabular}{|l|l|}
\hline Energy conversion efficiency $\eta$ & 0.8 \\
\hline Power Splitting Ratio $\rho$ & 0.5 \\
\hline Distance between CU and DT & $30 \mathrm{~m}$ \\
\hline Distance between CU and HAP & $40 \mathrm{~m}$ \\
\hline Distance between DT and HAP & $20 \mathrm{~m}$ \\
\hline Distance between DT and DR & $10 \mathrm{~m}$ \\
\hline Path-loss exponent $\alpha$ & 4 \\
\hline $\begin{array}{l}\text { Transmission rate requirement at the } \\
\text { D2D user } r_{d d}\end{array}$ & $0.5 \mathrm{bit} / \mathrm{Hz}$ \\
\hline $\begin{array}{l}\text { Maximum transmission power at the } \\
\text { HAP } P_{H, \text { max }}\end{array}$ & $1 \mathrm{~W}$ \\
\hline Noise Power $N_{0}$ & $10^{-7} \mathrm{~W}$ \\
\hline
\end{tabular}

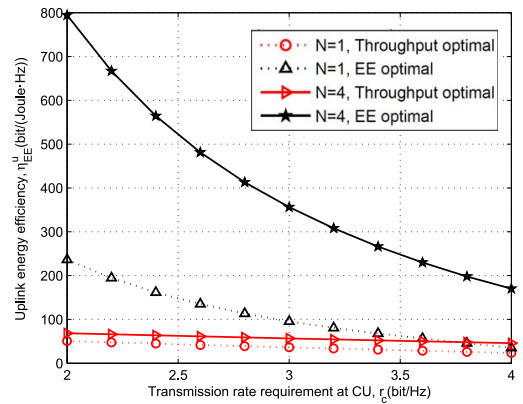

Fig. 2. Uplink energy efficiency v.s. transmission rate requirement at the CU.

\section{Performance Evaluation}

In this section, the performance of the proposed energyefficient WPCNs with cooperation transmissions is evaluated by the simulation experiments. For comparison, another two systems are also evaluated as the baselines. The one is the sum-throughput maximization, while the other one is the EE maximization with the aid of a single-antenna HAP.

The channel coefficient is represented by $\left(\frac{d_{i j}}{d_{0}}\right)^{-\frac{\alpha}{2}} \mathbf{h}$, where $\mathbf{h}$ is the Rayleigh distributed complex channel coefficient having a unit mean, $d_{i j}$ is the communication distance between nodes $i$ and $j$, the reference distance $d_{0}$ is 10 meters, and $\alpha$ is the path-loss exponent [15]. The simulation parameters are illustrated in Table I for the readers' convenience.

\section{A. System Energy Efficiency Versus CU's Transmission Rate Requirement}

In Fig. 2, we investigate the uplink energy efficiency versus transmission rate requirement of the $\mathrm{CU}$. We observe that the uplink energy efficiency of all the schemes decrease monotonically as $r_{c}$ increases. This is because the fact that a higher rate requirement results in more unreasonable energy consumption. Besides, we also see that the system achieves higher uplink energy efficiency when the HAP is equipped with multiple antennas. This reason is that the additional antennas can offer the extra degrees of freedom to conduct a more efficient WPT. Moreover, the proposed uplink EE-optimal scheme achieves at least a $200 \%$ higher energy efficiency than the throughput-optimal scheme. Fig. 3 shows the downlink

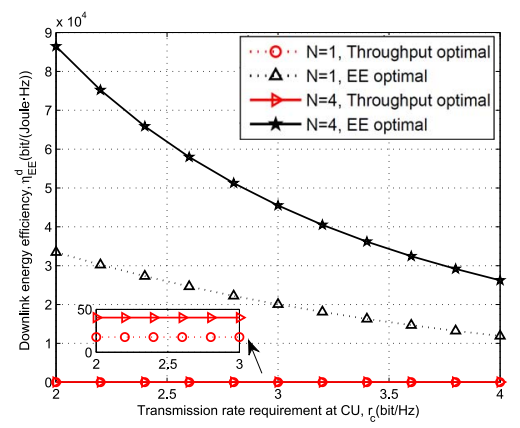

Fig. 3. Downlink energy efficiency v.s. transmission rate requirement at the $\mathrm{CU}$.

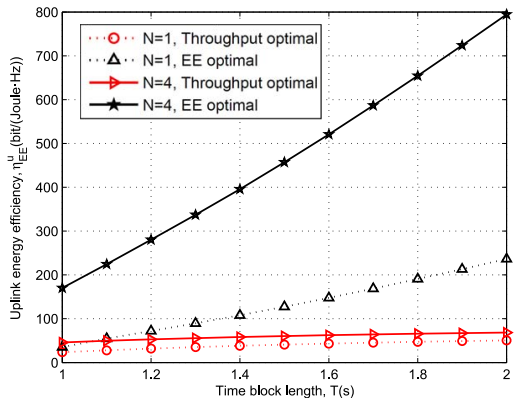

Fig. 4. Uplink energy efficiency v.s. time block length.

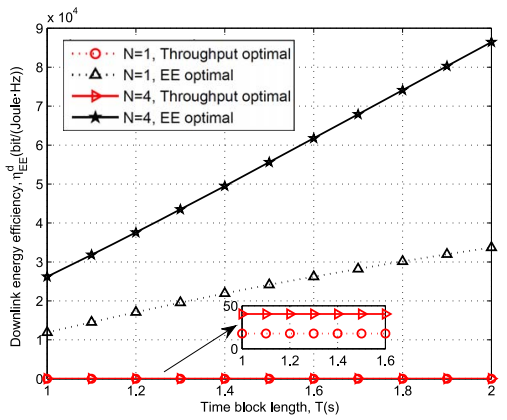

Fig. 5. Downlink energy efficiency v.s. time block length.

energy efficiency against CU's transmission rate requirement. Similar to the observation in uplink scenario, the proposed downlink EE-optimal scheme outperforms both of the baselines. Furthermore, we also observe from Figs. 2 and 3 that the proposed EE-optimal scheme is able to achieve higher EE gain than the throughput-optimal scheme, when CU's rate requirement is low. This is due to the fact that a larger rate requirement implies that the proposed EE-optimal system has to take more efforts to improve CU's transmission rate, which will inevitably reduce its EE gain compared to the throughput-optimal system.

\section{B. System Energy Efficiency Versus Time Block Length}

In Figs. 4 and 5, we display the uplink/downlink energy efficiency versus the time block length. It is shown that the uplink/downlink energy efficiency increases as the time block length increases. This is due to the fact that a longer time block length stands for a lower data rate requirement, which results in a more energy-efficient resource scheduling scheme. 


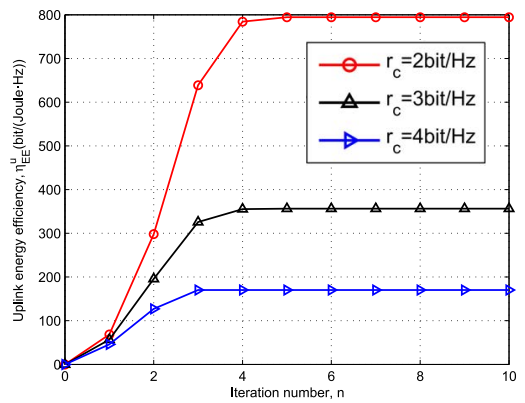

Fig. 6. Uplink energy efficiency v.s. iteration number.

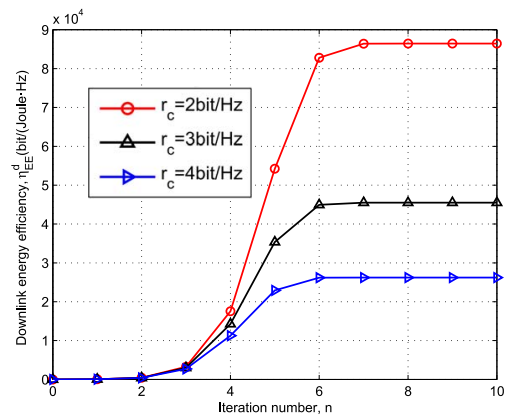

Fig. 7. Downlink energy efficiency v.s. iteration number.

Similarly, the proposed EE-optimal scheme achieves a great performance gain compared to those of the baseline schemes. Meanwhile, the proposed EE-optimal scheme exhibits higher EE-gain than the throughput-optimal scheme, when the time frame is long. Another important observation is that the EE under the downlink cooperation transmission is notably larger than that of the uplink scenario.

\section{Convergence of Proposed Algorithms}

Fig. 6 plots the convergence rate of the proposed uplink resource allocation strategy, i.e., Algorithms 1. We observe that it has a fast convergent rate and converges typically within 5 iterations. This is because the proposed Dinkelbach-type algorithm exhibits a super-linear convergence rate [26]. In Fig. 7, we show the convergence of the proposed downlink resource allocation scheme, i.e., Algorithm 2. We see that the Algorithm 2 can achieve convergence within 7 iterations and it converges almost simultaneously under different parameter settings. Furthermore, the uplink resource allocation algorithm converges faster than its downlink counterpart.

\section{CONCLUSION}

This paper studied the energy-efficient resource allocation and user cooperation protocol for wireless powered cellular networks (WPCNs). In particular, we maximized the uplink/downlink EE by jointly optimizing beamforming vector and the time domain resource allocation subject to the energy causality and the transmission rate requirements of users. Based on the fractional optimization theory and the classic SDR method, the original non-convex optimization problems were transformed into the corresponding convex problems. Furthermore, we proposed the energy-efficient resource allocation algorithms for the uplink and downlink transmissions and derived the optimal solution in semi-closed form by leveraging Lagrangian method. Simulation results revealed that the proposed algorithms converge to the optimal solutions within a few iterations and achieve notably higher EE compared to those of the baseline schemes.

\section{APPENDIX A}

PROOF OF THEOREM 2

Let $\left\{\boldsymbol{\tau}^{*}, \boldsymbol{\varepsilon}_{D}^{*}, R_{C}^{*}\right\}$ denote the optimal solution of problem (19). Then, we consider the following problem

$$
\begin{aligned}
& \max _{\mathbf{V}_{0}} R_{C}^{u *}+\tau_{4}^{*} \log \left(1+\frac{\left|\mathrm{g}_{D D}\right|^{2} \varepsilon_{D}^{(4) *}}{\tau_{4}^{*} N_{0}}\right)-u \operatorname{Tr}\left(\mathbf{V}_{0}\right) \\
& \text { s.t. } \mathrm{C} 11: \operatorname{Tr}\left(\mathbf{V}_{0}\right) \leq \tau_{1}^{*} P_{H, \max }, \\
& \mathrm{C} 12: \varepsilon_{D}^{(3) *}+\varepsilon_{D}^{(4) *} \leq \eta \operatorname{Tr}\left(\mathbf{H}_{D} \mathbf{V}_{0}\right), \\
& \mathrm{C} 13: R_{C}^{u *} \leq R_{C H}+R_{D H} \\
&=\tau_{2}^{*} \log \left(1+\frac{\eta\left|\mathrm{g}_{C H}\right|^{2} \operatorname{Tr}\left(\mathbf{H}_{C} \mathbf{V}_{0}\right)}{\tau_{2}^{*} N_{0}}\right) \\
&+\tau_{3}^{*} \log \left(1+\frac{\left|\mathrm{g}_{D H}\right|^{2} \varepsilon_{D}^{(3) *}}{\tau_{3}^{*} N_{0}}\right),
\end{aligned}
$$

$\mathrm{C} 14: R_{C}^{u *} \leq R_{C D}=\tau_{2}^{*} \log \left(1+\frac{\eta\left|\mathrm{g}_{C D}\right|^{2} \operatorname{Tr}\left(\mathbf{H}_{C} \mathbf{V}_{0}\right)}{\tau_{2}^{*} N_{0}}\right)$,

C15: $\mathbf{V}_{0} \succeq \mathbf{0}$.

Since $u>0$, the problem (38) is equivalent to

$$
\begin{array}{ll}
\min _{\mathbf{V}_{0}} & \operatorname{Tr}\left(\mathbf{V}_{0}\right) \\
\text { s.t. } & \mathrm{C} 16: \operatorname{Tr}\left(\mathbf{V}_{0}\right) \leq \tau_{1}^{*} P_{H, \max }, \\
& \mathrm{C} 17: \varepsilon_{D}^{(3) *}+\varepsilon_{D}^{(4) *} \leq \eta \operatorname{Tr}\left(\mathbf{H}_{D} \mathbf{V}_{0}\right), \\
& \text { C18: } \operatorname{Tr}\left(\mathbf{H}_{C} \mathbf{V}_{0}\right) \geq \gamma, \\
& \text { C19: } \mathbf{V}_{0} \succeq \mathbf{0} .
\end{array}
$$

$$
\begin{aligned}
& \underline{R_{C}^{u *}-\tau_{3}^{*} \log \left(1+\frac{\left|g_{D H}\right|^{2} \varepsilon_{D}^{(3) *}}{\tau_{3}^{*} N_{0}}\right)} \\
& \text { where } \gamma=\min \left\{\frac{\tau_{2}^{*} N_{0}\left(2 \longdiv { \tau _ { 2 } ^ { * } } - 1\right)}{\eta\left|g_{C H}\right|^{2}}\right. \text {, } \\
& \left.\frac{\tau_{2}^{*} N_{0}\left(2^{\frac{R_{C}^{u *}}{\tau_{2}^{*}}}-1\right)}{\eta\left|g_{C D}\right|^{2}}\right\} \text {. }
\end{aligned}
$$

Assuming that the problem (39) has an optimal solution $\mathbf{V}_{0}^{*}$, according to [40], we have

$$
\operatorname{Rank}^{2}\left(\mathbf{V}_{0}^{*}\right) \leq 3
$$

Since $\operatorname{Rank}\left(\mathbf{V}_{0}^{*}\right) \neq 0$, thus, $\operatorname{Rank}\left(\mathbf{V}_{0}^{*}\right)=1$. We construct a quintuple $\left\{\boldsymbol{\tau}^{*}, \boldsymbol{\varepsilon}_{D}^{*}, \mathbf{V}_{0}^{*}, R_{C}^{*}\right\}$ which satisfies all the constraints of problem (19) and achieves the maximum optimal value for problem (19). This ends the proof.

\section{APPENDIX B}

\section{PROOF OF THEOREM 3}

The partial KKT conditions are written as

$$
\begin{aligned}
\frac{\partial \mathcal{L}_{1}}{\partial \varepsilon_{D}^{(3)}} & =\frac{\alpha_{3}^{*} \tau_{3}^{*}\left|\mathbf{g}_{D H}\right|^{2}}{\ln 2\left(\tau_{3}^{*} N_{0}+\left|\mathbf{g}_{D H}\right|^{2} \varepsilon_{D}^{(3) *}\right)}-a_{2}^{*}=0 \\
\frac{\partial \mathcal{L}_{1}}{\partial \varepsilon_{D}^{(4)}} & =\frac{\left(1+a_{7}^{*}\right) \tau_{4}^{*}\left|\mathbf{g}_{D D}\right|^{2}}{\ln 2\left(\tau_{4}^{*} N_{0}+\left|\mathbf{g}_{D D}\right|^{2} \varepsilon_{D}^{(4) *}\right)}-a_{2}^{*}=0
\end{aligned}
$$




$$
\begin{aligned}
& \frac{\partial \mathcal{L}_{1}}{\partial \tau_{2}}=\left(a_{3}^{*}+a_{4}^{*}\right) \log \left(1+\frac{\eta\left|\mathbf{g}_{C D}\right|^{2} \operatorname{Tr}\left(\mathbf{H}_{C} \mathbf{V}_{0}^{*}\right)}{\tau_{2}^{*} N_{0}}\right) \\
& -\left(a_{3}^{*}+a_{4}^{*}\right) \frac{\frac{\eta\left|\mathbf{g}_{C D}\right|^{2} \operatorname{Tr}\left(\mathbf{H}_{C} \mathbf{V}_{0}^{*}\right)}{\tau_{2}^{*} N_{0}}}{\ln 2\left(1+\frac{\eta\left|\mathbf{g}_{C D}\right|^{2} \operatorname{Tr}\left(\mathbf{H}_{C} \mathbf{V}_{0}^{*}\right)}{\tau_{2}^{*} N_{0}}\right)} \\
& -a_{5}^{*}=0 \\
& \frac{\partial \mathcal{L}_{1}}{\partial \tau_{3}}=a_{3}^{*} \log \left(1+\frac{\left|\mathbf{g}_{D H}\right|^{2} \varepsilon_{D}^{(3) *}}{\tau_{3}^{*} N_{0}}\right) \\
& -a_{3}^{*} \frac{\frac{\left|\mathbf{g}_{D H}\right|^{2} \varepsilon_{D}^{(3) *}}{\tau_{3}^{*} N_{0}}}{\ln 2\left(1+\frac{\left|\mathbf{g}_{D H}\right|^{2} \varepsilon_{D}^{(3) *}}{\tau_{3}^{*} N_{0}}\right)} \\
& -a_{5}^{*}=0 \\
& \frac{\partial \mathcal{L}_{1}}{\partial \tau_{4}}=\left(1+a_{7}^{*}\right) \log \left(1+\frac{\left|\mathbf{g}_{D D}\right|^{2} \varepsilon_{D}^{(4) *}}{N_{0} \tau_{4}^{*}}\right) \\
& -\left(1+a_{7}^{*}\right) \frac{\frac{\left|g_{D D}\right|^{2} \varepsilon_{D}^{(4) *}}{N_{0} \tau_{4}^{*}}}{\ln 2\left(\frac{\left|g_{D D}\right|^{2} \varepsilon_{D}^{(4) *}}{N_{0} \tau_{4}^{*}}\right)}-a_{5}^{*}=0 \\
& a_{5}^{*}\left(T-\sum_{i=1}^{4} \tau_{i}\right)=0, a_{5}^{*} \geq 0, \sum_{i=1}^{4} \tau_{i} \leq T .
\end{aligned}
$$

After rearranging the Equations (41)-(46), we write the optimal solution $\left(\boldsymbol{\varepsilon}_{D}^{*}, \boldsymbol{\tau}^{*}\right)$ of (19) as given in Theorem 3 .

\section{APPENDIX C \\ PROOF OF THEOREM 5}

For the given $\left\{\boldsymbol{t}^{*}, \boldsymbol{\varepsilon}_{D}^{*}, R_{C}^{d *}\right\}$, the problem (31) is equivalent to

$$
\begin{array}{ll}
\min _{\mathbf{V}_{1}} & \operatorname{Tr}\left(\mathbf{V}_{1}\right) \\
\text { s.t. } & \text { C13': } \operatorname{Tr}\left(\mathbf{V}_{1}\right) \leq t_{1}^{*} P_{H, \max }, \\
& \text { C14': } \operatorname{Tr}\left(\mathbf{H}_{D} \mathbf{V}_{1}\right) \geq \beta_{1}, \\
& \text { C15': } \operatorname{Tr}\left(\mathbf{H}_{C} \mathbf{V}_{1}\right) \geq \beta_{2}, \\
& \text { C16': } \mathbf{V}_{1} \succeq \mathbf{0},
\end{array}
$$

where $\beta_{1}=\min \left\{\frac{\varepsilon_{D}^{(2) *}+\varepsilon_{D}^{(3) *}}{\eta \rho}, \frac{t_{1}^{*} N_{0}\left(2^{\frac{R_{*}^{d *}}{t_{1}^{*}}}-1\right)}{1-\rho}\right\}$ and $\beta_{2}=$ $t_{1}^{*} N_{0}\left(2 \frac{R_{C}^{d *}-t_{2}^{*} \log \left(1+\frac{\left|g_{C D}\right|^{2} \varepsilon_{D}^{(2)}}{t_{2}^{*} N_{0}}\right)}{t_{1}^{*}}-1\right)$. According to [40], we have

$$
\operatorname{Rank}^{2}\left(\mathbf{V}_{1}^{*}\right) \leq 3 .
$$

Since $\mathbf{V}_{1}^{*} \neq 0$, hence, $\operatorname{Rank}\left(\mathbf{V}_{1}^{*}\right)=1$.

\section{APPENDIX D}

\section{PROOF OF THEOREM 6}

The partial KKT conditions of problem (31) can be expressed as

$$
\begin{aligned}
& \frac{\partial \mathcal{L}_{2}}{\partial \varepsilon_{D}^{(2)}}=-b_{5}^{*}+\frac{b_{6}^{*}\left|\mathbf{g}_{C D}\right|^{2} t_{2}^{*}}{\ln 2\left(t_{2}^{*} N_{0}+\left|\mathbf{g}_{C D}\right|^{2} \varepsilon_{D}^{(2) *}\right)}=0 \\
& \frac{\partial \mathcal{L}_{2}}{\partial \varepsilon_{D}^{(3)}}=-b_{5}^{*}+\frac{\left(1+b_{3}^{*}\right)\left|\mathbf{g}_{D D}\right|^{2} t_{3}^{*}}{\ln 2\left(t_{3}^{*} N_{0}+\left|\mathbf{g}_{D D}\right|^{2} \varepsilon_{D}^{(3) *}\right)}=0
\end{aligned}
$$

$$
\begin{aligned}
\frac{\partial \mathcal{L}_{2}}{\partial t_{2}}= & -b_{1}^{*}+b_{6}^{*} \log \left(1+\frac{\left|\mathbf{g}_{C D}\right|^{2} \varepsilon_{D}^{(2) *}}{t_{2}^{*} N_{0}}\right) \\
& -\frac{b_{6}^{*}}{\ln 2} \frac{\frac{\left|\mathbf{g}_{C D}\right|^{2} \varepsilon_{D}^{(2) *}}{1+\frac{\left|\mathbf{g}_{C D}\right|^{2} \varepsilon_{D}^{(2) *}}{t_{2}^{*} N_{0}}}=0,}{\frac{\partial \mathcal{L}_{2}}{\partial t_{3}}=}-b_{1}^{*}+\left(1+b_{3}^{*}\right) \log \left(1+\frac{\left|\mathbf{g}_{D D}\right|^{2} \varepsilon_{D}^{(3) *}}{t_{3}^{*} N_{0}}\right) \\
& -\frac{\left(1+b_{3}^{*}\right)}{\ln 2} \frac{\mid \frac{\left|\mathbf{g}_{D D}\right|^{2} \varepsilon_{D}^{(3) *}}{t_{3}^{*} N_{0}}}{1+\frac{\left|\mathbf{g}_{D D}\right|^{2} \varepsilon_{D}^{(3) *}}{t_{3}^{*} N_{0}}}=0, \\
b_{1}^{*}\left(T-\sum_{i=1}^{3} t_{i}\right)= & 0, b_{1} \geq 0, \sum_{i=1}^{3} t_{i} \leq T .
\end{aligned}
$$

On rearranging the Equations (49)-(53), we deduce the optimal solution $\left(\varepsilon_{D}^{*}, t^{*}\right)$ as shown in Theorem 6 .

\section{REFERENCES}

[1] S. S. Kalamkar, J. P. Jeyaraj, A. Banerjee, and K. Rajawat, "Resource allocation and fairness in wireless powered cooperative cognitive radio networks," IEEE Trans. Commun., vol. 64, no. 8, pp. 3246-3261, Aug. 2016.

[2] J. An et al., "Achieving sustainable ultra-dense heterogeneous networks for 5G," IEEE Commun. Mag., vol. 55, no. 12, pp. 84-90, Dec. 2017.

[3] K. Xiong, C. Chen, G. Qu, P. Fan, and K. B. Letaief, "Group cooperation with optimal resource allocation in wireless powered communication networks," IEEE Trans. Wireless Commun., vol. 16, no. 6, pp. 3840-3853, Jun. 2017.

[4] J. Hu, K. Yang, G. Wen, and L. Hanzo, "Integrated data and energy communication network: A comprehensive survey," IEEE Commun. Surveys Tuts., vol. 20, no. 4, pp. 3169-3219, 4th Quart., 2018.

[5] G. Qiao, S. Leng, K. Zhang, and K. Yang, "Joint deployment and mobility management of energy harvesting small cells in heterogeneous networks," IEEE Access, vol. 5, pp. 183-196, 2017.

[6] S. Sudevalayam and P. Kulkarni, "Energy harvesting sensor nodes: Survey and implications," IEEE Commun. Surveys Tuts., vol. 13, no. 3, pp. 443-461, 3rd Quart., 2011.

[7] S. Mao, S. Leng, J. Hu, and K. Yang, "Power minimization resource allocation for underlay MISO-NOMA SWIPT systems," IEEE Access, vol. 7, pp. 17247-17255, 2019.

[8] Y. Liu, Z. Ding, M. Elkashlan, and H. V. Poor, "Cooperative nonorthogonal multiple access with simultaneous wireless information and power transfer," IEEE J. Sel. Areas Commun., vol. 34, no. 4, pp. 938-953, Apr. 2016.

[9] M. U. Kim and H. J. Yang, "Min-SINR maximization with DL SWIPT and UL WPCN in multi-antenna interference networks," IEEE Wireless Commun. Lett., vol. 6, no. 3, pp. 318-321, Jun. 2017.

[10] H. Chen, L. Xiao, D. Yang, T. Zhang, and L. Cuthbert, "User cooperation in wireless powered communication networks with a pricing mechanism," IEEE Access, vol. 5, pp. 16895-16903, 2017.

[11] Y. L. Che, L. Duan, and R. Zhang, "Spatial throughput maximization of wireless powered communication networks," IEEE J. Sel. Areas Commun., vol. 33, no. 8, pp. 1534-1548, Aug. 2015.

[12] H. Tabassum, E. Hossain, A. Ogundipe, and D. I. Kim, "Wirelesspowered cellular networks: Key challenges and solution techniques," IEEE Commun. Mag., vol. 53, no. 6, pp. 63-71, Jun. 2015.

[13] D. W. K. Ng and R. Schober, "Secure and green SWIPT in distributed antenna networks with limited backhaul capacity," IEEE Trans. Wireless Commun., vol. 14, no. 9, pp. 5082-5097, Sep. 2015.

[14] L. Tang, X. Zhang, P. Zhu, and X. Wang, "Wireless information and energy transfer in fading relay channels," IEEE J. Sel. Areas Commun., vol. 34, no. 12, pp. 3632-3645, Dec. 2016.

[15] P. Ramezani and A. Jamalipour, "Throughput maximization in dual-hop wireless powered communication networks," IEEE Trans. Veh. Technol., vol. 66, no. 10, pp. 9304-9312, Oct. 2017.

[16] H. Ju and R. Zhang, "Throughput maximization in wireless powered communication networks," IEEE Trans. Wireless Commun., vol. 13, no. 1 , pp. 418-428, Jan. 2014 
[17] W. Huang, H. Chen, Y. Li, and B. Vucetic, "On the performance of multiantenna wireless-powered communications with energy beamforming," IEEE Trans. Veh. Technol., vol. 65, no. 3, pp. 1801-1808, Mar. 2016.

[18] X. Kang, C. K. Ho, and S. Sun, "Full-duplex wireless-powered communication network with energy causality," IEEE Trans. Wireless Commun., vol. 14 , no. 10 , pp. 5539-5551, Oct. 2015.

[19] H. Ju and R. Zhang, "Optimal resource allocation in full-duplex wirelesspowered communication network," IEEE Trans. Commun., vol. 62, no. 10, pp. 3528-3540, Oct. 2014.

[20] H. Chen, Y. Li, J. L. Rebelatto, B. F. Uchôa-Filho, and B. Vucetic, "Harvest-then-cooperate: Wireless-powered cooperative communications," IEEE Trans. Signal Process., vol. 63, no. 7, pp. 1700-1711, Apr. 2015.

[21] H. Liang, C. Zhong, H. A. Suraweera, G. Zheng, and Z. Zhang, "Optimization and analysis of wireless powered multi-antenna cooperative systems," IEEE Trans. Wireless Commun., vol. 16, no. 5, pp. 3267-3281, May 2017.

[22] Q. Wu, G. Y. Li, W. Chen, and D. W. K. Ng, "Energy-efficient D2D overlaying communications with spectrum-power trading," IEEE Trans. Wireless Commun., vol. 16, no. 7, pp. 4404-4419, Jul. 2017.

[23] B. Fan, S. Leng, and K. Yang, "A dynamic bandwidth allocation algorithm in mobile networks with big data of users and networks," IEEE Netw., vol. 30, no. 1, pp. 6-10, Jan./Feb. 2016.

[24] G. Qiao, S. Leng, K. Zhang, and Y. He, "Collaborative task offloading in vehicular edge multi-access networks," IEEE Commun. Mag., vol. 56, no. 8, pp. 48-54, Aug. 2018.

[25] S. Buzzi et al., "A survey of energy-efficient techniques for 5G networks and challenges ahead," IEEE J. Sel. Areas Commun., vol. 34, no. 4, pp. 697-709, Apr. 2016.

[26] A. Zappone and E. Jorswieck, "Energy efficiency in wireless networks via fractional programming theory," Found. Trends Commun. Inf. Theory, vol. 11, nos. 3-4, pp. 185-396, Jun. 2015.

[27] J. Wu, S. Guo, J. Li, and D. Zeng, "Big data meet green challenges: Big data toward green applications," IEEE Syst. J., vol. 10, no. 3, pp. 888-900, Sep. 2016.

[28] Q. Wu, M. Tao, D. W. K. Ng, W. Chen, and R. Schober, "Energy-efficient resource allocation for wireless powered communication networks," IEEE Trans. Wireless Commun., vol. 15, no. 3, pp. 2312-2327, Mar. 2016.

[29] Q. Wu, W. Chen, D. W. K. Ng, J. Li, and R. Schober, "User-centric energy efficiency maximization for wireless powered communications," IEEE Trans. Wireless Commun., vol. 15, no. 10, pp. 6898-6912, Oct. 2016.

[30] Z. Zhou et al., "Energy-efficient stable matching for resource allocation in energy harvesting-based device-to-device communications," IEEE Access, vol. 5, pp. 15184-15196, 2017.

[31] B. Shang, L. Zhao, K.-C. Chen, and X. Chu, "Wireless-powered deviceto-device-assisted offloading in cellular networks," IEEE Trans. Green Commun. Netw., vol. 2, no. 4, pp. 1012-1026, Dec. 2018.

[32] K. Yang, S. Martin, C. Xing, J. Wu, and R. Fan, "Energy-efficient power control for device-to-device communications," IEEE J. Sel. Areas Commun., vol. 34, no. 12, pp. 3208-3220, Dec. 2016.

[33] G. Zhang, K. Yang, P. Liu, and J. Wei, "Power allocation for full-duplex relaying-based D2D communication underlaying cellular networks," IEEE Trans. Veh. Technol., vol. 64, no. 10, pp. 4911-4916, Oct. 2015.

[34] H. Kim, H. Lee, M. Ahn, H.-B. Kong, and I. Lee, "Joint subcarrier and power allocation methods in full duplex wireless powered communication networks for OFDM systems," IEEE Trans. Wireless Commun., vol. 15, no. 7, pp. 4745-4753, Jul. 2016

[35] Y. Zhang, J. Zhang, Y. Sun, and D. W. K. Ng, "Energy-efficient transmission for wireless powered D2D communication networks," in Proc. IEEE ICC, Paris, France, 2017, pp. 1-7.

[36] I. E. Nesterov and A. S. Nemirovskii, "Interior-point polynomial algorithms in convex programming," Stud. Appl. Math., vol. 13, 1994.

[37] J. Wu, S. Rangan, and H. Zhang, Green Communications: Theoretical Fundamentals, Algorithms, and Applications. Boca Raton, FL, USA: CRC press, 2012.

[38] S. Boyd and L. Vandenberghe, Convex Optimization. Cambridge, U.K.: Cambridge Univ. Press, 2004.

[39] R. M. Corless, G. H. Gonnet, D. E. Hare, D. J. Jeffrey, and D. E. Knuth, "On the Lambert W function," Adv. Comput. Math., vol. 5, no. 1, pp. 329-359, 1996.

[40] Y. Huang and D. P. Palomar, "Rank-constrained separable semidefinite programming with applications to optimal beamforming," IEEE Trans. Signal Process., vol. 58, no. 2, pp. 664-678, Feb. 2010.

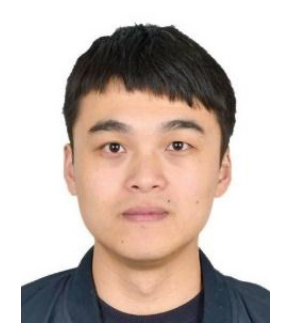

Sun Mao is currently pursuing the Ph.D. degree with the School of Information and Communication Engineering, University of Electronic Science and Technology of China. His research interests include simultaneous wireless information and power transfer, mobile edge computing, and cellular-connected UAV communications.

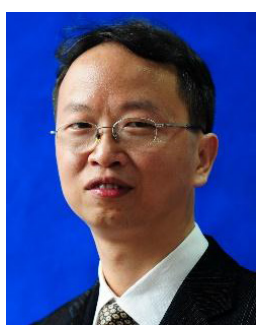

Supeng Leng received the Ph.D. degree from Nanyang Technological University, Singapore, where he was a Research Fellow with the Network Technology Research Center. He is currently a Professor with the School of Information and Communication Engineering, University of Electronic Science and Technology of China. He has authored or co-authored over 150 research papers in recent years. His research focuses on resource, spectrum, energy, routing, and networking in Internet of Things, vehicular networks, broadband wireless access networks, smart grid, and the next generation mobile networks. He serves as an Organizing Committee Chair and a TPC Member for many international conferences, as well as a Reviewer for over ten international research journals.

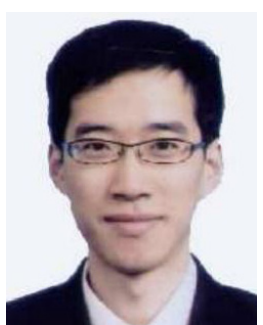

Jie Hu (S'11-M'16) received the B.Eng. and M.Sc. degrees from the Beijing University of Posts and Telecommunications, China, in 2008 and 2011, respectively, and the Ph.D. degree from the Faculty of Physical Sciences and Engineering, University of Southampton, U.K., in 2015. Since 2016, he has been with the School of Information and Communication Engineering, University of Electronic Science and Technology of China, China, as an Associate Professor. His current research is funded by the National Natural Science Foundation of China. He is also in great partnership with industry, such as Huawei and ZTE. He has served for ZTE Communications as the Guest Editor of the special issue "Wireless Data and Energy Integrated Communication Networks." He has a broad range of interests in wireless communication and networking, such as cognitive radio and cognitive networks, mobile social networks, data and energy integrated networks, as well as communication and computation convergence.

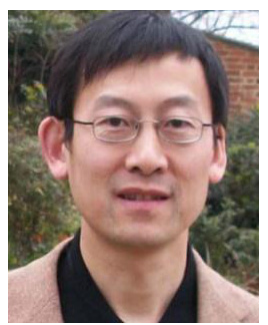

Kun Yang (SM'08) received the B.Sc. and M.Sc. degrees from the Computer Science Department, Jilin University, China, and the Ph.D. degree from the Department of Electronic and Electrical Engineering, University College London (UCL), U.K. He was with UCL on several European Union (EU) research projects for several years. In 2003, he joined the University of Essex, U.K., where he is currently a Chair Professor with the School of Computer Science and Electronic Engineering and also leading the Network Convergence Laboratory. $\mathrm{He}$ is also an Affiliated Professor with the University of Electronic Science and Technology of China, China. He manages research projects funded by various sources, such as U.K. EPSRC, EU FP7/H2020, and industries. He has published over 150 journal papers. His main research interests include wireless networks and communications, data and energy integrated networks, computation-communication cooperation. He serves on the editorial boards of both IEEE and non-IEEE journals. He has been a fellow of IET since 2009. 